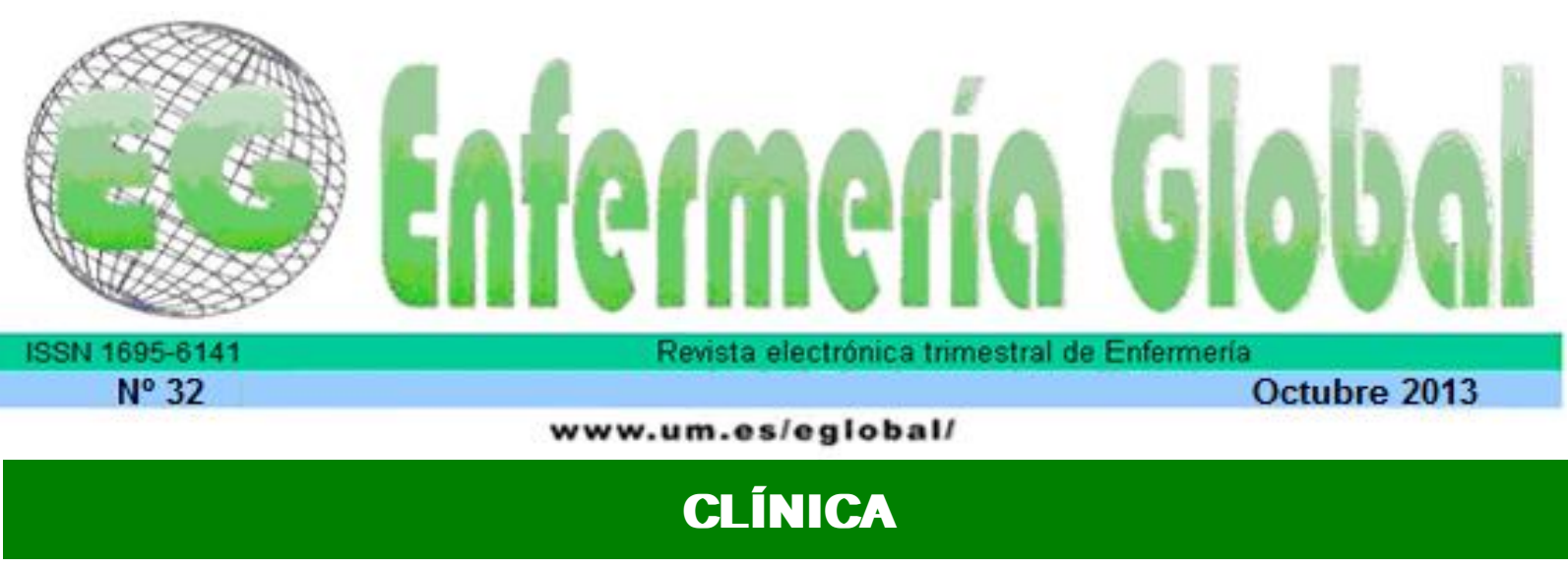

\title{
Prevalencia de diagnósticos de enfermería en personas con VIH/SIDA
}

Prevalence of nursing diagnoses in people with HIV/AIDS

\section{*Gómez Gómez, Janny Juliana *Mayorga Calderón, Mª Emilce *Pérez Moreno, José Orlando *Rojas Sánchez, Lyda Zoraya *Orozco Vargas, Luis Carlos *Camargo Figuera, Fabio Alberto.}

\author{
*Grupo de Investigación GRINFER, Escuela de Enfermería, Universidad Industrial de Santander, \\ Bucaramanga, Colombia. E-mail: falcamfi@uis.edu.co
}

Palabras clave: diagnóstico de Enfermería; VIH, SIDA; prevalencia. Keywords: Nursing diagnosis; HIV; AIDS; Prevalence.

\section{RESUMEN}

Introducción: La infección por el Virus de la Inmunodeficiencia Humana y el Síndrome de Inmunodeficiencia Adquirida se ha convertido en un problema de salud pública, es por ello que el cuidado de enfermería de las personas con esta enfermedad debe estar dirigido a los problemas reales y potenciales más frecuentes y prioritarios.

Objetivo: Determinar la prevalencia de los diagnósticos de enfermería en personas con VIH/SIDA que reciben atención ambulatoria en instituciones de salud de Bucaramanga, Colombia.

Métodos: Se realizó un estudio de corte transversal en adultos con VIH/SIDA que recibían atención ambulatoria en algunas instituciones de salud de Bucaramanga.

Resultados: Se encontraron prevalencias para 79 diagnósticos, con un mínimo de 4 y un máximo de 46 diagnósticos por persona. Seis diagnósticos presentaron una prevalencia mayor del $50 \%$, que fueron: riesgo de infección y riesgo de lesión con 98,9\%, seguido por protección inefectiva con $96,7 \%$, riesgo de deterioro de la integridad cutánea con $95,6 \%$, deterioro del patrón del sueño con $57,1 \%$ y sedentarismo con $53,8 \%$.

Conclusiones: Esta investigación es una propuesta para que enfermería estandarice la atención brindada y sus planes de cuidado en las personas que viven con VIH/SIDA basándose en el proceso de enfermería y en el uso de lenguaje estandarizado. Se hace imperativa la necesidad de investigación en esta área con estudios sobre los diagnósticos más prevalentes. 


\section{ABSTRACT}

Introduction: Infection with Human Immunodeficiency Virus and Acquired Immune Deficiency Syndrome has become a public health problem, so the nursing care of people with this disease should be directed to the real problems and to the most common and main potentials.

Objective: To determine the prevalence of nursing diagnoses in people with HIV / AIDS receiving outpatient care in health institutions in Bucaramanga, Colombia.

Methods: A cross-sectional study in adults with HIV / AIDS who were receiving outpatient care in some health institutions in Bucaramanga.

Results: Prevalences were found for 79 diagnoses, with a minimum of 4 and a maximum of 46 diagnoses per person. Six diagnoses had a prevalence greater than $50 \%$, which were risk of infection and risk of injury with $98.9 \%$, followed by ineffective protection with $96.7 \%$, risk of impaired skin integrity with $95.6 \%$, impaired sleep pattern $57.1 \%$ and $53.8 \%$ sedentary lifestyle.

Conclusions: This research is a proposal to standardize the care provided nursing and care plans for people living with HIV / AIDS based on the nursing process and the use of standardized language. It is imperative the necessity for research in this area with studies of high prevalence.

\section{INTRODUCCIÓN}

La infección por el Virus de la Inmunodeficiencia Humana (VIH) y el Síndrome de Inmunodeficiencia Adquirida (SIDA) se ha convertido en una problema de salud pública. De acuerdo con los datos reportados por el Programa Conjunto de las Naciones Unidas sobre el VIH/SIDA (ONUSIDA) ${ }^{(1)}$ se estima que en el mundo 40,3 millones de personas viven con el $\mathrm{VIH}$ y que durante el año 2005, cerca de cinco millones contrajeron la infección. Para América Latina, se calcula que alrededor de 1,8 millones de personas están viviendo con el VIH. En Colombia se puede apreciar una tendencia a la generalización de la epidemia y según modelos predictivos para el año 2005, basados en los resultados de estudios centinela realizados en el país, se puede estimar que alrededor de 170 mil personas entre los 15 y los 49 años viven con $\mathrm{VIH} / \mathrm{SIDA}$ en Colombia. El Síndrome de Inmunodeficiencia Adquirida tiene importantes repercusiones sociales, que abarcan todos los ámbitos y que para el sector salud le corresponde proporcionar cuidados adecuados a estas personas, esto ha supuesto un importante aumento de las preocupaciones prácticas y éticas ${ }^{(2)}$.

La gestión de estrategias ideadas para mejorar la calidad de vida y evolución de las personas con enfermedades crónicas como el VIH/SIDA debe dirigirse a los problemas reales y potenciales más comunes en este tipo de personas. Enfermería cuenta con dos herramientas, el lenguaje estandarizado y el proceso de enfermería, en el cual el diagnóstico es el eje central, porque los problemas que identifica son la base del plan de cuidado y además son determinantes para definir las intervenciones efectivas que logren los resultados de las cuales es responsable el profesional de enfermería ${ }^{(3)}$.

En el ámbito nacional no se encontraron estudios publicados que presentaran la prevalencia de los diagnósticos de enfermería en personas con VIH/SIDA y en el ámbito mundial la bibliografía al respecto es escasa, por lo cual la enfermera ante el $\mathrm{VIH} / \mathrm{SIDA}$, esta desafiada a determinar un cuidado específico, efectivo y describirlo en un lenguaje estandarizado ${ }^{(4)}$. 
Recientemente, se han realizado estudios que han determinado los diagnósticos de enfermería en personas con enfermedades infecciosas hospitalizadas ${ }^{(5)}$ y en personas que viven con VIH/SIDA en servicios ambulatorios ${ }^{(6)}$, destacando la necesidad de realizar estudios con lenguaje enfermero en personas con VIH/SIDA, permitiendo así mejorar el cuidado, eligiendo las intervenciones más adecuadas de acuerdo a las condiciones de cada persona cuidada.

Por tal razón, el objetivo del presente estudio fue determinar los diagnósticos de enfermería más frecuentes en personas con VIH/SIDA y de esta manera proporcionar herramientas a enfermería para planear y ejecutar intervenciones que logren los resultados propuestos y mejoren la calidad de vida de estas personas.

\section{MÉTODOS}

Se realizó un estudio de corte transversal descriptivo. La población de estudio estuvo conformada por personas con VIH/SIDA atendidas en servicios ambulatorios y de consulta externa de instituciones de salud de la ciudad de Bucaramanga (Colombia), entre enero y marzo de 2009. El tamaño de la muestra fue de 91 personas que fueron seleccionadas de acuerdo a su aceptación de participar en el estudio. Con dicho tamaño de muestra se esperaba detectar, con un poder del $80 \%$, un nivel de confianza del $95 \%$ y una precisión del 10\%, diagnósticos de enfermería con prevalencias iguales o mayores a $10 \%$. Los criterios de inclusión fueron todas las personas adultas mayores de 18 años con VIH/SIDA que acudieron a los servicios de consulta externa de instituciones de salud de Bucaramanga. Se excluyeron mujeres embarazadas y personas con alteración en la esfera mental, con alteraciones crónicas o graves que no le permitían responder la entrevista y adultos con limitaciones de la comunicación.

El instrumento utilizado fue el "Formato de valoración de tamizaje del adulto", el cual fue validado por un grupo investigador de la Escuela de Enfermería de la Universidad Industrial de Santander (UIS), cuenta con validez facial, de contenido, de criterio, reproducibilidad y además incluye las categorías diagnosticas de la NANDA (Diagnósticos Enfermeros, Definiciones y clasificaciones) $2001-2002{ }^{(7)}$, formato que fue adaptado para los objetivos de la presente investigación. Se realizó una prueba piloto con 10 personas atendidas en un hospital de tercer nivel de atención por consulta externa, a partir de la cual se realizaron modificaciones a algunas preguntas poco comprensibles por los individuos entrevistados. La entrevista se realizó en personas ambulatorias atendidas en dos instituciones de apoyo de primer nivel atención y en un hospital de tercer nivel atención, ambas pertenecientes al área metropolitana de la ciudad de Bucaramanga, Colombia. La entrevista fue aplicada por los mismos investigadores, el tiempo aproximado por entrevista fue de 45 minutos.

Se utilizaron 111 variables por cada persona entre esas 96 categorías diagnósticas de la NANDA 2001-2002 con sus características definitorias principales. Se tuvo en cuenta el criterio que Lynda Carpenito considera como requisito para la determinación del diagnóstico de enfermería. Para las categorías diagnósticas de riesgo, que aún no han sido clasificadas por Lynda Juall Carpenito, se trabajó con la presencia de al menos un factor de riesgo basándonos en los factores de riesgo de la nueva versión de la NANDA $2007-2008^{(8)}$, y para las categorías diagnósticas reales se consideró que el diagnóstico estuvo presente cuando se le identifica al menos una característica definitoria principal ${ }^{(9)}$. 
La información recolectada se insertó en una base de datos doble e independiente en el programa Epi Info $6.04 d^{(10)}$ y la confrontación se realizó por medio del subprograma VALIDATE de Epi Info, posteriormente se hicieron correcciones de errores y se exportaron los datos para su análisis a Stata $10^{(11)}$.

Se realizó un análisis descriptivo de las características generales de la muestra donde en las variables cualitativas se presentaron las frecuencias absolutas y relativas, las variables cuantitativas con medidas de dispersión y tendencia central. Se calcularon las prevalencias de los diagnósticos de enfermería con intervalos de confianza del 95\% y se determinó el mínimo y el máximo número de diagnósticos por persona.

La investigación se realizó de acuerdo a la resolución No. 08430 de 1993 del Ministerio de Salud de Colombia, por la cual se establecen las normas científicas, técnicas y administrativas para la investigación en salud ${ }^{(12)}$. El presente estudio no ofreció ningún tipo de riesgo para los participantes, por lo cual el consentimiento informado se obtuvo de forma verbal. En él se garantizó la confidencialidad de la información y su desarrollo se orientó bajo el respeto de los principios éticos de autonomía, beneficiencia, no maleficencia y justicia.

\section{RESULTADOS}

La edad promedio de las personas entrevistadas fue de 36,9 años, con una desviación estándar de 10,1 años, con edades entre 18 y 66 años; el promedio de años de escolaridad fue de 8,6 años, con una desviación estándar de 3,8 y un rango entre 0 y 16 años; el 53,8\% $(n=49)$ correspondía al género masculino; la mayoría de ellos no conviven con pareja 70,3\% ( $n=64)$; de estratos bajos $76,9 \%(n=70)$; seguridad social el $61,5 \%(n=56)$ pertenece al régimen subsidiado, $19,7 \%(n=18)$ al régimen contributivo, $15,3 \%(n=14)$ al SISBEN y el 3,3\% $(n=3)$ son vinculados; el $87,91 \%$ $(n=80)$ corresponden a personas de instituciones de salud de primer nivel de atención (Instituciones de apoyo) y el $12,1 \%(n=11)$ a una institución de tercer nivel de atención.

En el estudio se incluyeron 96 categorías diagnósticas de enfermería de las cuales se encontró prevalencia a 79, ver Tabla I. Del total de los diagnósticos 6 tienen prevalencia mayor del 50\%, que fueron: riesgo de infección y riesgo de lesión, seguido por protección inefectiva, riesgo de deterioro de la integridad cutánea, deterioro del patrón del sueño y sedentarismo. 
Tabla I. Prevalencias de los diagnósticos de enfermería mayores al 10\%.

\begin{tabular}{lcc}
\hline \multicolumn{1}{c}{ ETIQUETA DIAGNÓSTICA } & PREVALENCIA & IC 95\% \\
\hline 1. Riesgo de lesión & 98 & $94-99$ \\
2. Riesgo de infección & 98 & $94-99$ \\
3. Protección inefectiva & 96 & $90-99$ \\
4. Riesgo deterioro de la integridad & 95 & $89-98$ \\
5. Deterioro del patrón del sueño & 57 & $46-67$ \\
6. Sedentarismo & 53 & $43-64$ \\
7. Conductas generadoras de salud & 40 & $30-51$ \\
8. Conflicto de decisiones & 32 & $23-43$ \\
9. Deterioro en el mantenimiento del & 32 & $23-43$ \\
10. Dolor & 32 & $23-43$ \\
11. Deterioro de la dentición & 30 & $20-40$ \\
12. Riesgo de automutilación & 27 & $18-37$ \\
13. Riesgo de autolesión & 25 & $16-35$ \\
14. Trastorno de la imagen corporal & 25 & $16-35$ \\
15. Náuseas & 24 & $15-34$ \\
16. Fatiga & 22 & $13-31$ \\
17. Desesperanza & 19 & $12-29$ \\
18. Impotencia & 18 & $11-28$ \\
19. Riesgo de soledad & 17 & $10-27$ \\
20. Baja autoestima situacional & 17 & $10-27$ \\
21. Riesgo de intolerancia a la & 17 & $10-27$ \\
22. Desequilibrio nutricional por defecto & 17 & $10-27$ \\
23. Disfunción sexual & 15 & $8-24$ \\
24. Déficit de actividades recreativas & 15 & $8-24$ \\
25. Desequilibrio nutricional por exceso & 15 & $8-24$ \\
26. Interrupción de los procesos & 14 & $7-23$ \\
27. Afrontamiento defensivo & 14 & $7-23$ \\
28. Dolor crónico & 14 & $7-23$ \\
29. Deterioro de la adaptación & 12 & $6-20$ \\
30. Riesgo de deterioro del rol parental & 12 & $6-20$ \\
31. Aislamiento social & 12 & $6-20$ \\
32. Deterioro de la interacción social & 12 & $6-20$ \\
33. Duelo anticipado & 12 & $6-20$ \\
34. Duelo disfuncional & 11 & $5-19$ \\
35. Incapacidad del adulto para & 11 & $5-19$ \\
36. Intolerancia a la actividad & 11 & $5-19$ \\
\hline & & \\
& &
\end{tabular}

Los diagnósticos de menor prevalencia fueron déficit de volumen de líquidos, hipertermia, perfusión tisular inefectiva periférica, incontinencia urinaria de estrés, estreñimiento subjetivo, déficit de autocuidado: vestido-acicalamiento, déficit de autocuidado: uso del baño y conflicto del rol parental con $1,1 \%$. No se encontró prevalencia para los diagnósticos: riesgo de desequilibrio de la temperatura corporal, hipotermia, riesgo de aspiración, deterioro de la deglución, incontinencia urinaria funcional, retención urinaria, riesgo de incontinencia urinaria de urgencia, incontinencia urinaria refleja, desatención unilateral, disreflexia autónoma, riesgo de disreflexia autónoma, déficit de autocuidado: alimentación, déficit de autocuidado: baño/higiene, deterioro de la movilidad en silla de ruedas, deterioro de la habilidad para la traslación, deterioro de la movilidad en la cama y riesgo de disfunción neurovascular periférica. 
El número de diagnósticos de enfermería mínimo que presentó una persona fue de 4 y el máximo de 46 . El $50,5 \%$ de la personas tienen presentes 110 menos diagnósticos y el restante porcentaje tienen presentes más de 11 diagnósticos de enfermería.

\section{DISCUSIÓN}

Con el presente estudio se logró determinar la frecuencia de los diagnósticos de enfermería en personas que viven con VIH/SIDA atendidas en servicios ambulatorios, obteniendo así los diagnósticos más prevalentes en esta población.

En un estudio de diagnósticos de enfermería en personas hospitalizadas basadas en los signos y síntomas médicos documentados en la historia clínica se hallaron 45 diferentes diagnósticos de enfermería, por persona se encontraron de 1 a 9 diagnósticos (13); en el presente estudio se encontraron prevalencias para 79 diagnósticos, con un mínimo de 4 y un máximo de 46 diagnósticos por persona.

En la revisión bibliográfica realizada se encontraron tres estudios (13-15) de diagnósticos de enfermería en personas con VIH/SIDA hospitalizados, pero al hacer la comparación con estos tres estudios, ver Tabla II, se debe tener en cuenta que la presente investigación fue realizada en personas con VIH/SIDA de servicios de consulta externa y ambulatorios, similar al estudio de Cunha y Galvão ${ }^{(6)}$. 
Tabla II. Comparación con otros estudios acerca de la prevalencia de los diagnósticos de enfermería en personas con VIH/SIDA.

\section{Diagnósticos de enfermería}

\begin{tabular}{|c|c|c|c|c|c|}
\hline Número de participantes en cada estudio & $16^{*}$ & 98 & 60 & 51 & 91 \\
\hline $\begin{array}{l}\text { Alteración de la nutrición: menos que las } \\
\text { exigencias del cuerpo (Desequilibrio nutricional } \\
\text { por defecto) }\end{array}$ & 81 & - & - & 23.5 & 17.6 \\
\hline $\begin{array}{l}\text { Diagnósticos respiratorios: Limpieza inefectiva } \\
\text { de las vías aéreas/ Patrón respiratorio ineficaz/ } \\
\text { Deterioro del intercambio gaseoso }\end{array}$ & 81 & 25.5 & 85 & - & 9.9/5.5/7.7 \\
\hline Riesgo/Deterioro de la integridad cutánea & 75 & - & 86.6 & 11.7 & $95.6 / 5.5$ \\
\hline Diarrea & 69 & - & - & 7.8 & 9.9 \\
\hline Riesgo de infección & 62.5 & 55 & 100 & 100 & 98.9 \\
\hline Dolor (Dolor agudo/Dolor crónico) & 56 & - & - & - & $32.9 / 14.3$ \\
\hline Aislamiento social & 56 & - & - & 9.8 & 12.1 \\
\hline Afrontamiento ineficaz & 44 & - & - & - & 9.9 \\
\hline Ansiedad/Temor & 44 & 76.5 & $90 / 83$ & $1.9 / 1.9$ & 4.4 \\
\hline Intolerancia a la actividad/Fatiga & 44 & - & 93.3 & 3.9 & $11 / 22$ \\
\hline Déficit de conocimientos & 32 & 99 & 88.3 & 19.6 & 7.7 \\
\hline Sufrimiento espiritual & 25 & - & - & - & 2.2 \\
\hline Hipertermia & 25 & - & - & - & 1.1 \\
\hline Riesgo de lesión & 25 & 45 & 38.8 & - & 98.9 \\
\hline Déficit del autocuidado & 25 & 26.5 & - & 39.2 & 1.1 \\
\hline Déficit de volumen de líquidos & - & 11 & - & - & 1.1 \\
\hline Alteración de la perfusión tisular & - & 5 & - & - & 1.1 \\
\hline Duelo anticipado & - & 1 & - & - & 12.1 \\
\hline Protección inefectiva & - & - & 100 & - & 96.7 \\
\hline Deterioro del patrón del sueño & - & - & 100 & 25.4 & 57.1 \\
\hline Disfunción sexual & - & - & - & 66.6 & 15 \\
\hline Deterioro de la dentición & - & - & - & 1.9 & 30 \\
\hline Baja autoestima situacional & - & - & - & 1.9 & 17 \\
\hline
\end{tabular}

*Número de planes de cuidado.

De lo observado en la Tabla II, tenemos que las prevalencias entre los estudios de paciente ambulatorios son muy similares, mientras que las diferencias con los estudios de pacientes hospitalizados son mayores.

Al realizar la comparación de nuestros resultados con el estudio de Silva y colaboradores ${ }^{(15)}$, hay gran similitud en los diagnósticos riesgo/deterioro de la integridad cutánea, riesgo de infección y protección inefectiva con prevalencias por encima del $80 \%$. Se esperaba una alta prevalencia de estos diagnósticos por el comportamiento propio del problema de salud crónico que padecen estas personas (VIH/SIDA), en el cual hay una alteración de las defensas secundarias primordialmente (linfocitos CD4, monocitos y macrófagos) y la aparición de algunas enfermedades oportunistas que afectan las defensas primarias (lesiones de la piel).

Las instituciones de donde se obtuvo la muestra de este estudio brindan educación continua sobre estilos de vida saludable, haciéndose evidente el impacto de sus 
intervenciones en la baja prevalencia de los diagnósticos de enfermería relacionados con el autocuidado. En este sentido encontramos que se reporta una prevalencia cercana al $26 \%$ en personas hospitalizadas por dos estudios $(14,15)^{13,14}$, en nuestro trabajo encontramos una frecuencia mucho menor para personas ambulatorias, cerca del $1 \%$. $Y$ este contraste es aun más notorio para el diagnóstico déficit de conocimientos que presentó una prevalencia del $99 \%$ (13) ${ }^{13}$ en personas hospitalizadas y en personas ambulatorias de $7.7 \%$ para el presente estudio, similar al $19.6 \%$ del estudio de Cunha y Galvão ${ }^{(6)}$. En cuanto a diagnósticos de alteración de la nutrición diferente a lo reportado en un estudio con personas hospitalizadas donde presentó una prevalencia del $81 \%(13)^{14}$, en el presente estudio se reporta una frecuencia de $17.6 \%$.

En este estudio se encontró un diagnóstico de bienestar: Conductas Generadoras de salud, con una prevalencia de 40,6\%, que indica que el apoyo de las instituciones de salud es de gran importancia para estas personas, ya que su principal objetivo es promover hábitos saludables.

También los diagnósticos y preocupaciones psicosociales identificadas en este estudio están presentes en los estudios anteriores; diagnósticos como ansiedad, temor, desesperanza, depresión, aislamiento social y otros están presentes en ambos grupos con prevalencias similares, estos resultados son apoyados en la literatura clínica según Smith ${ }^{(13,14)}$, la estigmatización y discriminación por el VIH/SIDA se sigue manteniendo, hay que continuar trabajando en el desarrollo de estrategias diseñadas a combatir el estigma y la exclusión social de este grupo de personas, desarrollando marcos legales necesarios para proteger los derechos y la dignidad de la personas que padecen esta enfermedad.

Es de resaltar que en el presente estudio se utilizó un formato de valoración general para la recolección de los datos que fue diseñado y evaluado por un grupo de investigadores de la Escuela de Enfermería de la Universidad Industrial de Santander en el año 2001 y además se hizo una valoración focalizada individualmente realizada por enfermeros. En algunos de los artículos encontrados no hay suficiente claridad sobre estos aspectos, la identificación de los diagnósticos de enfermería se realizó por información obtenida de los signos y síntomas registrados en la historia clínica médica.

\section{CONCLUSIÓN}

Enfermería tiene como objeto el fomento y cuidado de la salud en las personas. Para lograrlo se requiere de una interacción dinámica entre la persona de cuidado, familia y la enfermera; con esta investigación hemos logrado identificar las fortalezas y debilidades de la personas con VIH/SIDA, el reto de la enferma ante esta situación es utilizar los recursos disponibles en la familia y comunidad, planificar acciones con miras a obtener los resultados esperados, es decir, a disminuir la prevalencia de los diagnósticos encontrados que sin duda repercuten en la calidad de vida de las personas que conviven VIH/SIDA.

Finalmente, esta investigación permitió determinar los diagnósticos de enfermería más frecuentes en personas con VIH/SIDA y es una propuesta para que los profesionales de enfermería estandaricen el cuidado en las personas con VIH/SIDA basándose en el proceso de enfermería y en los diagnósticos más prevalentes en esta población. 
Es claro que el cuidado de enfermería se debe basar en un plan de cuidados estandarizado que permita ofrecer resultados con calidad y eficiencia, que repercutan en el bienestar físico, psicológico y mental de las personas con VIH/SIDA, ya establecidos los diagnósticos de enfermería más prevalentes en esta población, se requiere su posterior validación, lo que permitirá a los profesionales de enfermería establecer claridad conceptual y un lenguaje estandarizado a la hora de elaborar los planes de cuidado. Ya que sin consenso sobre los problemas de las personas con $\mathrm{VIH} / \mathrm{SIDA}$, es imposible comunicarse de manera efectiva sobre el cuidado de enfermería en estas personas.

De esta manera se hace imperativa la necesidad de investigación en esta área con la realización de estudios hacia la validación clínica de estos diagnósticos ${ }^{(16,17)}$ y de ensayos clínicos controlados ${ }^{(18-22)}$, que evalúen el efecto de las intervenciones de enfermería sobre los diagnósticos más prevalentes de este estudio.

\section{REFERENCIAS BIBLIOGRÁFICAS}

1. Porras de Quintana L, Tamayo-Buendía M, Sánchez-Vanegas G. Prevalencia de Infección por VIH en Pacientes con Patología Dermatológica en un Centro de Referencia Nacional 2005-2006. Rev. salud pública 2009;11(4): 613-619.

2. Tomás-Sábado J, Aradilla A. Actitud ante el SIDA en estudiantes de enfermería. ¿Cuál es el papel de la formación académica? Educ Méd 2003;6:87-92

3. NANDA, Diagnósticos enfermeros: definiciones y clasificación 2005-2006. Elseiver España. 2005, Madrid-España.

4. Kristine A, Davis MA. Aids nursing care and standardized sursing language: an application of the nursing intervention classification. ANAC 1995; 6(6).

5. Neves JF, Paixão FRC, Lima TC, Ceolim MF. Nursing diagnoses of patients admitted for Infectious Diseases. Acta paul. enferm. 2010;23(6): 818-823.

6. Cunha GH, Galvão MTG. Diagnósticos de enfermagem em pacientes com o Vírus da Imunodeficiência Humana/ Síndrome da Imunodeficiência Adquirida em assistência ambulatorial. Acta paul. enferm. 2010; 23(4): 526-532.

7. Orozco LC, Cañas Duran M, Céspedes VM, Matajira Rondón G, Mogollón Niño YC. Validación del formato de valoración de Tamizaje del adulto. Universidad Industrial de Santander, 2002.

8. NANDA, Diagnósticos enfermeros: definiciones y clasificación. Elseiver 2008, Madrid-España.

9. Carpenito LJ. Diagnosticos de enfermería. Aplicaciones a la práctica clínica. 9a edición. MacGraw-Hill, Interamericana. 2003.

10. CDC. Epi Info, v. 6.04d - StatCalc. Epidemiología en ordenadores. Atlanta, Georgia. Enero, 2001.

11.StataCorp. Stata Statistical Sofware: Release 10. Collage Station, Texas: Stata Corporation; 2008

12. Ministerio de salud. República de Colombia. Resolución número 008430 del 4 de octubre de 1993.

13. Smith AR. Examination of nursing diagnoses for adults hospitalized with acquired immunodeficiency syndrome, 1982-1990. Nurs Diagn. 1991;2(3):1118.

14.Smith AR, Chang BL. Nursing diagnoses for hospitalized patients with AIDS. Nurs Diagn. 1996 Jan-Mar;7(1):9-18.

15.Silva Myria Ribeiro da, Bettencourt Ana Rita de Cássia, Diccini Solange, Belasco Angélica, Barbosa Dulce Aparecida. Diagnósticos de enfermagem em 
portadores da Síndrome da Imunodeficiência Adquirida. Rev. bras. enferm. 2009;62(1):92-99.

16. Carrillo González GM, Rubiano Mesa YL. La investigación en validación de diagnósticos de enfermería. Rev Cubana Enfermer 2007;23(3). Disponible en: http://bvs.sld.cu/revistas/enf/vol23_3_07/enf09307.html

17. Pinilla-Gómez E, Orozco-Vargas LC, Camargo-Figuera FA, Berrío JA, MedinaMoreno LX. Bullying en adolescentes escolarizados: validación del diagnóstico de enfermería "riesgo de violencia dirigida a otros". Hacia Promoc Salud 2012;17(1):45-58.

18.Bohórquez-Bueno VC, Castañeda-Pérez BA, Trigos-Serrano LJ, OrozcoVargas LC, CamargoFiguera FA. Eficacia de la intervención enfermera para cuidadores con cansancio del rol del cuidador. Rev Cubana Enfermer 2011; 27(3) 210-219

19.Pinilla GE, Cáceres FdM , Orozco-Vargas LC. Gradiente dosis respuesta de las intervenciones de enfermería para el control del temor en gestantes. Rev Cubana Enfermer 2010;26(2)27-36.

20.Rojas-Sánchez OA, Rueda-Díaz LJ, Vesga-Gualdrón LM, Orozco-Vargas LC, Forero Bulla CM, Camargo-Figuera FA. Eficacia de las intervenciones de enfermería para el diagnóstico "manejo inefectivo del régimen terapéutico" Enferm Clin 2009; 19(6):299-305.

21. Orozco-Vargas LC, Rangel R, Lopez LE, Rueda E, Ortiz AF. Eficacia de las intervenciones de Enfermería del Temor pre-quirúrgico. Un Ensayo clínico controlado. Actualizaciones en Enfermería 2005; 8(1):8-13.

22. Cáceres Manrique FM, Bohórquez Caballero F, Puerto Pedraza HM, Smith Hernández D. Eficacia de las intervenciones de enfermería para disminuir el temor en personas que inician tratamiento para el cáncer. Un ensayo clínico controlado. Med Unab 2004; 7(21):161-165. 